\title{
Análisis descriptivo de un proceso de implementación de Aprendizaje Basado en Proyectos en Educación para el Desarrollo y Cooperación Internacional
}

\author{
Dra. Begoña Mora Jaureguialde', Manuel Jesús Hermosín \\ Mojeda ${ }^{2}$
}

Resumen: El presente artículo expone un estudio de corte longitudinal realizado en el contexto de una asignatura universitaria a través de un proceso de innovación continuada que se ha llevado a cabo durante cinco cursos académicos para incorporar competencias profesionales no abordadas desde los contenidos académicos. Desde la asignatura de Educación para el Desarrollo y la Cooperación Internacional de Educación Social en la Universidad de Huelva se realizan proyectos socioeducativos que impliquen un conocimiento del Tercer Sector y que promuevan una ciudadanía global crítica y comprometida que lidere valores sociales en los desempeños profesionales. Se muestra el trabajo realizadocon con más de 500 alumnos que han producido durante 5 cursos más de 100 proyectos socioeducativos. Con el trabajo desempeñado los resultados arrojan que esta metodología es la adecuada para abordar este tipo de contenidos competenciales de corte social.

Palabras clave: Educación para el desarrollo; ciudadanía global; proyectos socioeducativos; educación superior.

\footnotetext{
' Licenciada en Psicopedagogía y Dra. en Educación. Profesora Ayudante Doctora. Área de Didáctica y Organización Escolar. Departamento de Pedagogía. Facultad de Educación, Psicología y CC. del Deporte. Universidad de Huelva.

${ }^{2}$ Licenciado en Filosofía y CC. de la Educación. Profesor Asociado Área de Teoría e Historia de la Educación. Departamento de Pedagogía. Facultad de Educación, Psicología y CC. del Deporte. Universidad de Huelva.
} 
Fecha de recepción: 15 de marzo de 2020.

Fecha de admisión definitiva: 26 de junio de 2020 .

\section{Descriptive analysis of a project-based learning implementation process in De- velopment Education and International Cooperation}

Abstract: This article presents a longitudinal study perfomed in the context of a university subject over a period of five academic years by means of a process of continuous innovation. Its aim was to incorporate professional skills traditionally not provided by the academic content of the subject. The Social Education degree from the University of Huelva includes socio-educational projects carried out in the context of the subject of Education for Development and International Cooperation. These projects provide a deeper understanding of the third sector and foster a critical and committed global citizenship promoting social values in professional activities. The article shows the work accomplished with the help of more than 500 students, who have completed more than 100 socio-educational projects over a period of five academic years. After sucessful completion, the results show that this methodology is adequate when the goal is to address this type of contents relating to social competences.

Key words: Education for development; global citizenship; social-educational projects, higher education.
Analyse descriptive d'un processus de mise en œuvre de l'apprentissage basé sur des projets dans le domaine de l'éducation au développement et de la coopération internationale

Résumé: Cet article présente une étude longitudinale réalisée dans le cadre d'une discipline universitaire sur une période de cing années académiques au moyen d'un processus $d$ 'innovation continue. Son objectif était $\mathrm{d}^{\prime}$ intégrer des compétences professionnelles qui n'étaient traditionnellement pas fournies par le contenu académique de la matière. Le diplôme en Éducation Sociale del'Université de Huelva comprend des projets socio-éducatifs réalisés dans le cadre du sujet de l'Éducation au Développement et de la Coopération Internationale. Ces projets permettent de mieux connaître le troisième secteur et de favoriser une citoyenneté mondiale critique et engagée en promouvant les valeurs sociales dans les activités professionnelles. L'article montre le travail accompli grâce à la collaboration de plus de 500 étudiants, qui ont réalisé plus de 100 projets socio-éducatifs sur une période de cinq années universitaires. Les résultats montrent que cette méthodologie est adéquate lorsque l'objectif est d'aborder ce type de contenus relatifs aux compétences sociales.

Mots clé: Éducation au développement, citoyenneté mondiale, projets socio-éducatifs, enseignement supérieur. 


\section{Introducción}

Los retos propuestos a la universidad con la incorporación al Espacio Europeo de Educación Superior (EEES), nos han proporcionado oportunidades para modificar los paradigmas más tradicionales de la enseñanza superior universitaria española. Lejos van quedando las clases expositivas gracias a la incorporación de metodologías de enseñanza experimentadas en décadas pasadas que habiendo demostrado su eficacia, dejaron de utilizarse por comodidad, por imperativo institucional o por desidia del docente: Ya indicaba en el pasado siglo Vilar (1997, p.11-16), que los métodos resultaban "simplistas y rígidos, cuando hemos empezado a construir la sociedad posindustrial: la sociedad de la información y del conocimiento". Si la sociedad que se intentaba crear era una sociedad

que se inspira en las investigaciones e invenciones de la física cuántica [...]; que incluye en constructivismo piagetiano; la que se fundamenta en la biología y en la sociología centradas en la auto-organización; la que critica la economía tradicional; la de la historia que interroga al pasado para responder al presente y el futuro [...]; la de las neurociencias que coordinan sus trabajos con las ciencias de la cognición y la nueva generación de las máquinas informáticas; la nueva racionalidad es, en suma, la de los científicos de todas las disciplinas (Vilar, 1997, p. 11-16).

Dentro de este contexto de futuro es donde planteamos el objetivo principal de este trabajo, dar a conocer un proceso de buenas prácticas pedagógicas en el aula, que ha permitido mejorar el aprendizaje del alumnado gracias a la elaboración de proyectos, método que ha facilitado transformar su conciencia individual y pensar en cooperación y en desarrollo con un sentido de responsabilidad, con la capacidad de relacionar causas y efectos, de manera que se descubran nuevas formas de trabajar, crecer y desarrollarnos y desarrollar. Para conseguirlo, se solicita al alumnado la elaboración de proyectos que intercedan por países, zonas geográficas o poblaciones determinadas, con la única finalidad de mejorar las condiciones vitales en general, o de minimizar una situación conflictiva en particular, desde la educación para el desarrollo.

Para explicar la experiencia, se abordarán algunas de las metodologías activas más utilizadas para profundizar en el trabajo por proyectos en la universidad. Seguidamente se plantea la propuesta de trabajo a través de los proyectos de carácter internacional dentro de la asignatura, ahondando en los objetivos que se les plantean, la metodología llevada a cabo a través del enfoque del marco lógico. Se incorporan los resultados obtenidos por los estudiantes para concluir con el análisis del proceso metodológico realizado, los resultados y las conclusiones. 


\section{Metodologías activas en la universidad}

Hace décadas que se incorporaron satisfactoriamente en niveles educativos previos a la Universidad y con notable éxito, diversas maneras de comprender el enseñar y aprender, tal y como muestran numerosas publicaciones (Baro, 2011 ; Hernández, 2000; Mérida et al, 2011; Parrilla, Muñoz-Cadavid y Sierra, 2013); sin embargo, modificar la tan arcaica y constreñida estructura universitaria cuesta tiempo y esfuerzo. Si bien actualmente podemos afirmar que se ha conseguido traspasar esa frontera y la institución superior está valiéndose de herramientas facilitadoras, no solo la adquisición de contenidos de forma memorística, sino que permiten desarrollar "la capacidad de análisis, de síntesis o la evaluación de alternativas y toma de decisiones", como dicen Almagro y Pérez (2004, p. 23), gracias a la incorporación de metodologías activas.

Rodríguez, Ramírez y Fernández (2017, p. 80) afirman que

la corriente pedagógica constructivista tan popular hoy, está basada en la teoría del conocimiento que aborda el aprendizaje en el desarrollo de la autonomía del estudiante, dando al docente un papel observador y poco interventor.

Así, la construcción del aprendizaje produce que nos centremos en Aprender haciendo y se pierde de vista la importancia de la compresión de los contenidos. Según Lazarri (2014), las metodologías activas son utilizadas como recurso para mantener la atención del alumnado, aunque no explicita qué tipo de metodología concreta sería la efectiva, ya que existen muchas variaciones, por ejemplo, al aprendizaje basado en problemas, donde una investigación diagnóstica indagaría en los conceptos previos de los docentes y discentes para resolver problemas; a los métodos de casos, donde hay un aprendizaje activo y cooperativo junto con los estudiantes a través de una situación real; al aprendizaje basado en proyectos, donde el protagonista del proceso de enseñanza-aprendizaje es el alumnado y el profesorado pasa a ser orientador; al aprendizaje cooperativo o colaborativo, en el que surge un sistema relacional donde los componentes del grupo se organizan e influyen recíprocamente y a tantos otros (Rodríguez, Ramírez y Fernández, 2017, p. 82).

Como incentivo, y para facilitar la incorporación de esta corriente innovadora a las aulas universitarias, son numerosas las publicaciones que parten de experiencias concretas que ayudan a sistematizar estas experiencias para darlas a conocer, y así permitir una mayor utilización de metodologías dinámicas, lo que favorece el aprendizaje individual del alumnado (Orejudo, Fernández y Garrido, 2008; Rodríguez, Ramírez y Fernández, 2017). Como ejemplo, las que se llevan a cabo 
en las universidades de la Comunidad Valenciana en el ámbito del AprendizajeServicio o los Trabajos Fin de Máster universitario de Cooperación al Desarrollo de dichas universidades (U. de València, U. Jaume I, U. Miguel Hernández, U. Politécnica de Valencia y U. de Alacant) donde se pueden poner en práctica -en contextos reales-, los aprendizajes teóricos del aula. Todas estas experiencias y otras, de otros contextos, nos sirven de ejemplo para llevar a cabo nuestra experiencia, aunque con un enfoque diferente.

Si bien se podría haber elegido cualquiera de las estructuras pedagógicas nombradas anteriormente, en el estudio que se presenta, se selecciona el Aprendizaje Basado en Proyectos, ya que como afirma Bell (2010), un proceso de este tipo se puede resumir en cuatro fases: primero, determinar qué se quiere investigar (plantear una pregunta); segundo, definir los conceptos fundamentales y realizar las averiguaciones necesarias; tercero, elaborar el proyecto que permita mostrar y compartir lo aprendido; $y$, por último, exponer el proyecto al resto de compañeros. Con esta premisa, se plantea como una de las metodologías fundamentales en el currículum de la asignatura de $3^{\circ}$ de carrera de la Titulación de Grado en Educación Social de la Universidad de Huelva, denominada Educación para el Desarrollo (EpD) y la Cooperación Internacional (Cl).

\section{I. Los proyectos de trabajo como método de aprendizaje}

Desde que iniciara su andadura en la segunda mitad del siglo XX, en la Facultad de Medicina de la Universidad de McMaster (Canadá), el aprendizaje basado en proyectos (en adelante ABP) ha ido ganando terreno en el planteamiento de muchas asignaturas de corte distinto en el ámbito universitario, tratando de conjugar, en su uso, el desarrollo de competencias, puesto que "el enfoque comunitario se plantea una educación que dé respuestas a las circunstancias de la realidad social" (Parrilla, Muñoz-Cadavid y Sierra, 2013, p. 16).

En la Universidad de Huelva, como en el resto de las universidades españolas, gracias a la implantación del EEES, se ha producido un cambio sustancial en el diseño de los programas de las asignaturas, incluyéndose, en dichas planificaciones, las competencias que deben adquirir los alumnos al finalizarlas o bien, al desarrollo de qué competencias contribuyen las diferentes asignaturas que se incluyen en un plan de estudios para facilitar el desempeño del profesional tras su paso por la academia. Las competencias se convierten, por tanto, en el elemento fundamental en la formación universitaria y podemos definirlas como apuntan Alonso, Cruz y Hermosín (2010) como la capacidad de un sujeto de aplicar en el momento más 
idóneo la estrategia o conocimiento más oportuno. No solo implica el dominio del conocimiento o de estrategias o procedimientos, sino también la capacidad o habilidad de saber cómo utilizarlo en el momento más adecuado.

Estas aproximaciones tienen sentido cuando entendemos un rol de centro educativo, no centrado exclusivamente en lo que acontece en el interior de las aulas, sino de lo que sucede fuera de esas paredes institucionales. Si bien se debe tener en consideración que los modelos relacionales serán un reflejo de la relación escuela-comunidad, es por ello que la sociedad está demandando una apertura de los centros educativos al medio (Parrilla, Muñoz-Cadavid y Sierra, 2013).

Si lo que se pretende es el desarrollo de las competencias básicas del estudiantes del siglo XXI -capacidad de análisis, síntesis, evaluación de alternativas y toma de decisiones-, el uso de metodologías activas se torna fundamental (Almagro y Pérez, 2004).

Desafortunadamente, en la educación superior ha primado la transmisión de contenidos teóricos sobre las materias que está estudiando, sin embargo, probablemente tenga pocas referencias sobre la forma de aplicar ese contenido a la situación práctica real que se le presentará en el futuro laboral. Por estos motivos, la razón principal por la que optamos por este enfoque metodológico del $A B P$ es porque permite crear grupos de trabajo en los que los alumnos y alumnas deben manejar los contenidos que van adquiriendo para desarrollar un producto final donde conjuguen -con la mayor similitud a un contexto real-, las estrategias para dar respuesta a la situación que origina el proyecto. Según Krajcik y Blumenfeld (2006), el ABP pretende construir el conocimiento en función de los siguientes planteamientos:

- Los estudiantes deben construir el conocimiento activamente de acuerdo a sus interacciones y experiencias con el mundo. El profesorado no proporcionará todos los contenidos a adquirir, sino que facilitará que los estudiantes observen, interactúen con el fenómeno, adquieran nuevas ideas y las relaciones con lo que ya aprendieron.

- El alumnado debe sumergirse en un contexto auténtico y cercano al mundo real, y especialmente útil para adquirir el conocimiento eficazmente. Los estudiantes suelen valorar más y mejor la importancia de las tareas que deben realizar y se espera un incremento de la capacidad de generalización con respecto a otras situaciones similares. 
- El ABP fomenta el trabajo cooperativo entre estudiantes, profesores y otros miembros de la comunidad. Trabajar juntos en un mismo proyecto mejora la construcción del conocimiento por el aporte que puede hacer cada uno desde sus distintas experiencias y conocimientos.

- El ABP favorece la puesta en práctica de las herramientas cognitivas porque ellas pueden expandir y amplificar lo que los estudiantes pueden aprender. Las nuevas tecnologías mejoran aún más las capacidades de los estudiantes al ser incluidas en el ABP.

Como se ha dejado entrever, el Aprendizaje Basado en Proyectos

es una metodología de aprendizaje en la que se pide a los alumnos que, en pequeños grupos, planifiquen, creen y evalúen un proyecto que responda a las necesidades planteadas en una determinada situación (SIEUPM, 2008, p. 4).

Así, el alumnado con un "papel más activo, como protagonista de su aprendizaje" (Orejudo, Fernández y Garrido, 2008, p. 23), debe situar el contexto de actuación en la aplicación del conocimiento teórico adquirido en la asignatura que cursa. El proyecto que desarrollarán es guiado por el docente, quien tutela los grupos de trabajo y orienta sus procesos de toma de decisiones. En la asignatura que nos ocupa el trabajo por proyectos encaja perfectamente ya que según Vergara Ramírez (2014),

en el contexto del aprendizaje del siglo XXI, el análisis de los procesos globales necesita estrategias didácticas que inviten a las personas a reflexionar sobre su propia realidad, su contexto, su identidad y su posición en la comunidad donde habitan: el centro educativo, su familia, sus amigos, su comunidad física y su red virtual (p. 150).

En el ámbito de trabajo de los educadores sociales, la realización de proyectos suele ser una de las tareas fundamentales para actuar en muchos contextos; especialmente, en el de las ONGDs u otro tipo de asociaciones o colectivos dedicados al Desarrollo y a la Cooperación ya sea de carácter nacional o internacional.

Los organismos que gestionan las ayudas oficiales al desarrollo promueven el funcionamiento mediante la financiación de proyectos $y$, en el ámbito privado, por su parte, las fundaciones y empresas que entre sus fines sociales tienen el soporte económico de actividades con fines solidarios, convocan la presentación de proyectos igualmente, para canalizar de esa forma su participación en dichas actividades.

En este contexto descrito la preparación del alumnado en realización de proyectos es fundamental como parte de su formación de educadores y educadoras sociales 
ya que como apuntan Ortega Carpio, Sianes Castaño y Cordón Pedregosa (2012), se debe tener en cuenta

la importancia capital del profesorado, ya no exclusivamente como legatario y transmisor de conocimientos, sino como promotor y garante de este proceso educativo holístico. Es el profesorado el que, mediante su compromiso activo, está llamado a procurar el éxito en el proceso de ED a nivel universitario, gracias a la tutorización y al acompañamiento que puede brindar a sus estudiantes (p. 618).

De esta forma, el equipo docente responsable de la asignatura, conocedor de esta realidad, incorpora en los créditos prácticos de la asignatura, el aprendizaje basado en proyectos, lo que pasamos a detallar a continuación.

\section{La Propuesta de Trabajo: ABP en Educación para el Desa- rrollo y la Cooperación Internacional}

Tras realizar una revisión de procedimientos existentes en materia de comprensión de los proyectos de trabajo, vamos a exponer algunas de las cuestiones que podría caracterizar al trabajo realizado en el aula a través de los proyectos de trabajo según Hernández (2000):

1. Un recorrido por un tema-problema que favorece el análisis, la interpretación y la crítica (como contraste de puntos de vista). Partiremos de un tema sugerido por el alumno si bien necesitará orientación del docente; lo importante es encontrar una temática motivadora para ser explorada.

2. Predomina la actitud de cooperación y el profesor es un aprendiz y no un experto (pues ayuda a aprender sobre temas que ha de estudiar con los alumnos). Trabajar por proyectos supone un aprendizaje para ambos actores del proceso.

3. Un recorrido que busca establecer conexiones entre los fenómenos que cuestionan la idea de una versión única de la realidad. No hay una única verdad en los fenómenos educativos.

4. Cada recorrido es singular y se trabaja con diferentes tipos de información. No existen fórmulas mágicas que poder aplicar repetidamente.

5. El docente enseña a escuchar. Se pueden incorporar conversaciones, entrevistas, debates y su análisis formará parte del contenido del proyecto, se observará al otro como facilitador del propio aprendizaje. 
6. Lo que queremos enseñarles hay diferentes formas de aprenderlo ly no sabemos si aprenderán eso u otras cosas). A pesar de que a través de la evaluación se pretende determinar el grado de aprendizaje de cada alumno, la realidad es que dentro de una clase cada estudiante tiene un ritmo y un estilo de aprendizaje diferente, de ahí que no se pueda afirmar lo que se aprenderá al final del proceso.

7. Un acercamiento actualizado a los problemas de las disciplinas y los saberes. La selección de los temas de los proyectos se encuentra mediatizada por la cultura de la organización del currículum, si bien se anima a salirse de los estereotipos habituales.

8. Una forma de aprendizaje en la que se tiene en cuenta que todos los alumnos pueden aprender si encuentran el lugar par ello. Facilitaría el que cada alumno pueda encontrar su lugar en el grupo.

9. No se olvida que el aprendizaje vinculado al hacer, a la actividad manual y a la intuición, también es una forma de aprendizaje. Se presta atención a la forma, al modo en el que se presenta el recorrido realizado por el tema o problema (Hernández, 2000, pp. 47-50).

\section{I. Objetivo}

Si bien nuestro objetivo principal, al presentar este trabajo, se resume en dar a conocer las buenas prácticas pedagógicas desarrolladas en el aula gracias a la incorporación de metodologías activas en la docencia universitaria, centradas en procesos de enseñanza-aprendizaje a través de proyectos, hemos planteado otros objetivos operativos que nos permiten que el alumnado transforme su conciencia individual y entienda la cooperación y el desarrollo desde un sentido de responsabilidad social, con la capacidad de relacionar causas y efectos, de manera que se descubran nuevas formas de trabajar, de crecer y de desarrollarse:

1. Establecer actividades donde se fomente el uso del aprendizaje reflexivo (Proyectos de Educación para el Desarrollo en contexto internacional).

2. Ofrecer al alumnado las herramientas necesarias para facilitarles la selección de temáticas en las que centrar sus propuestas de proyectos de cooperación y desarrollo formativo. 
3. Analizar situaciones concretas derivadas de la diversidad (cultural, geográfica, social...) con el fin de tenerlas en cuenta para establecer criterios específicos en el diseño de proyectos de educación para el desarrollo.

4. Diseñar tareas que desarrollen el pensamiento analítico del alumnado, capacitándolo para identificar lo esencial de lo secundario y para tomar conciencia de las consecuencias de las decisiones tomadas.

5. Partir de un análisis de la realidad lo más exhaustivo posible para proponer soluciones formativas viables en los casos objeto de estudio.

6. Promover procesos de evaluación formativa donde el alumnado participe de manera activa: autoevaluación, coevaluación y heteroevaluación.

\subsection{Metodología}

Durante la implantación de estos procesos de enseñanza activa han participado 140 grupos de estudiantes de la titulación de Grado en Educación Social durante cinco cursos académicos (541 estudiantes en total), lo que nos ha permitido hacer un estudio longitudinal de la propuesta que presentamos. Esta propuesta se inscribe en un marco más amplio donde se incluye el uso de metodologías activas variadas gracias a los años de experiencia del profesorado implicado y a su participación en las diferentes convocatorias de proyectos de innovación docente de la Facultad de Educación, Psicología y Ciencias del Deporte de la Universidad de Huelva.

En este caso nos centramos en la aplicación del ABP partiendo de un caso concreto que cada grupo debe seleccionar, crear o modificar en función de los criterios que se dan en clase, tal como se expone en los objetivos. Concretamente el procedimiento seguido es el siguiente: en el aula se divide al alumnado en grupos de entre 3 y 6 participantes, donde cada uno de ellos debe analizar situaciones cuasi reales, experiencias propias $\mathrm{u}$ otras experiencias cercanas relacionadas con las necesidades educativas que plantea la diversidad cultural, entendida como "cultura contemporánea, una cuestión fundamental para que un individuo pueda comprender el mundo en el que vive es que sepa cómo acceder, analizar e interpretar la información" (Hernández, 2000, p. 46). El profesorado da una serie de indicaciones para facilitar la elaboración del caso del tema a estudiar:

- La selección del tema es resultado de la totalidad de las preferencias de temáticas de cada uno de los componentes del grupo, por lo que deben llegar a 
un consenso después de plantearse cuál es el problema principal que deben solucionar desde instancias educativas.

- Los casos deben ser internacionales, poniendo a España en relación con cualquier país y teniendo en cuenta las líneas directrices de la Agencia de Cooperación Española (países y áreas preferentes, según el Plan Director vigente). También cabe la posibilidad de que pueda ser considerada la intervención a realizar en nuestro país, pero en ese caso los destinatarios serían extranjeros.

- El proyecto, de temática libre, debe centrarse en la educación para el desarrollo y la cooperación internacional cumpliendo, por lo tanto, un perfil socioeducativo y donde se pueda promover la ciudadanía global.

- En aras de una visión sostenible del proyecto, se establece como criterio a considerar preferentemente la actuación, apoyo y/o comunicación con una entidad (ONGD, Asociación...) que actúe en el país de implementación del proyecto y que trabaje en la temática seleccionada o cuyas líneas de intervención estén relacionadas con el proyecto.

- Otro criterio fundamental es el conocimiento de la legislación en materia de cooperación al desarrollo de España y de la del país donde se va a presentar el proyecto, como marcos fundamentales para poder implementar cualquier intervención de cooperación internacional al desarrollo.

- El diseño de los proyectos debe ser factible, abierto y que posibiliten la discusión. En cada caso y según la situación, se deberán reflejar diferentes transversalidades: género, respeto al medio ambiente, la atención a la diversidad y la inclusión educativa.

Se inicia el trabajo determinando la temática a abordar mediante una discusión grupal, técnica de investigación que podría servir para cualquier tipo de estudio. Delgado y Gutiérrez (1999, p. 354) definen el grupo de discusión como

un dispositivo utilizable para la facilitación de la tarea de enseñanza-aprendizaje individual en situación de grupo particularmente para inducir o facilitar la motivación individual hacia el aprendizaje..., tratándose de una de las técnicas fundamentales en la investigación social.

Hecho esto-quizá lo más complicado-, se pasa a la elección del país, para lo que, en principio, solo se proponen las limitaciones anteriormente expuestas. El principal escollo en este tema puede estar en obtener y entender la normativa vigente en 
materia del proyecto que quieran desarrollar y que se incluirá en el análisis de la realidad previo a la formulación del proyecto.

Una vez que se tiene el grupo social, de edad, etc., con el que se trabajará y el lugar donde se va a realizar, país, localidad, barrio, asociación, etc., se pasa a la descripción del caso en sí y su contextualización. Este punto deberá ser lo más detallada posible, incluyendo la necesidad educativa, formativa, del caso planteado, por tratarse de intentar solucionar problemas sociales desde el punto de vista educativo.

A partir de ese momento, toda la energía del alumnado se centrará en dar solución a ese caso, analizándolo, desgranándolo, siguiendo los pasos establecidos por Navarro (2011, p. 11):

1. Lectura y análisis del caso individualmente: el estudiante debe plantearse preguntas básicas sobre el caso asumiendo el papel de protagonista y tratando de responder a preguntas como ¿Qué haría yo si me encontrara en esta situación?

2. Análisis y discusión del caso en pequeño grupo: este es el momento de discutir y poner en común aquello que se ha trabajado individualmente, se trata de consensuar el enfoque del caso y proponer alternativas para la intervención.

3. Discusión del caso con el profesorado: las propuestas consensuadas de cada grupo de trabajo son compartidas con el profesorado y los grupos han de estar preparados para responder a las cuestiones sobre el caso, que se les plantee.

El análisis de los casos se centra en la claridad en la selección y exposiciones de las necesidades detectadas en el grupo objeto de estudio, bien sea a nivel educativo, social, sanitario, estructural, comunitario, etc. En los grupos de trabajo se incorporan desde la suposición, la situación tanto del sujeto o grupo objeto de estudio, como del entorno, comunidad, país.

Una cuestión determinante que se valora en el proceso es la viabilidad del proyecto, de esta forma intentamos que el aprendizaje sea lo más competencial posible. El grupo de estudiantes debe identificar a una o varias instituciones/asociaciones de ambos países que trabajen en el área, como contraparte, y usuarios específicos seleccionados para permitir el trabajo colaborativo entre ambas entidades. Se pide algo tan concreto para que tomen conciencia de la necesidad de trabajar con todos los derechos en el país en el que ubican la intervención, cumpliendo normativas y leyes locales, así como facilitar en el caso de impedimento de desplazamiento, la 
ejecución del proyecto de intervención educativa en el propio país con medios y recursos locales. Se pretende que sea factible su implementación.

Como todo proyecto socioeducativo habrá una serie de dificultades con los que se pueden encontrar la hora de llevar a cabo la ejecución del proyecto. Para ello se propone la evaluación y el seguimiento del proceso. Se plantea la necesidad de evaluar el proceso formativo, el proyecto en sí, y a los actores de dicho proceso, de forma que se realiza una evaluación múltiple, si bien coordinada, como dice Navarro (2011 , p. 12), "se podría realizar la evaluación, abordando para ello la evaluación inicial, continua y final. Hemos contemplado las técnicas de evaluación a utilizar tales como la observación, listas de control o los registros individuales y evaluación conjunta entre profesionales considerando así a todos los profesionales implicados en la intervención".

\subsection{Cómo abordar el trabajo: El Enfoque del Marco Lógico}

Con los casos planteados y las posibles temáticas a abordar encima de la mesa, es momento de comenzar a plantear la respuesta estructura a través de uno de los documentos que serán objeto de evaluación. Para ello se les plantea que realicen el proyecto según el enfoque del marco lógico (EML), cuyos principios y estructura pasamos a relatar a continuación.

El marco lógico muestra las reglas para la planificación del proyecto de una forma en que la descripción de éste sea objetiva, los resultados sean fácilmente evaluables y su redacción esté claramente estructurada. Este enfoque

es una herramienta metodológica utilizada en la Planificación de Proyectos Orientada por Objetivos cuyos orígenes la sitúan en la elaboración de proyectos estratégicos estadounidenses de gran envergadura (Ferrero y de Loma-Osorio, 2008, p. 61).

Sus principales características desde el punto de vista de la contribución a la mejora de la calidad de la gestión de los proyectos de desarrollo son: (Gómez y Sainz, 2013, p. 101)

- El EML constituye, en primer lugar, un método para tomar decisiones. Esto no es un rasgo original y exclusivo del mismo, ya que todos los instrumentos de planificación son sistemas para la toma de decisiones.

- El EML mantiene esencialmente un enfoque participativo en el que, de manera especial, se integran las personas y colectivos afectados por la futura intervención de desarrollo. 
Los elementos básicos para los proyectos desde esta perspectiva del Marco Lógico son:

1. El objetivo general.

2. Los objetivos específicos.

3. Los resultados esperados.

4. Las actividades necesarias para alcanzar dichos resultados.

5. Los recursos necesarios para desarrollar las actividades.

6. Las limitaciones externas del proyecto.

7. Los indicadores medibles y objetivos para evaluar el proyecto.

8. El procedimiento para determinar los indicadores.

Según Ferrero y de Loma-Osorio (2008, p. 64), la descripción de los elementos del proyecto sería:

- El OBJETIVO GLOBAL (OG) es el principal objetivo general al cual se quiere que contribuya el proyecto a largo plazo.

- EI OBJETIVO ESPECÍFICO(OE) establece la situación que se espera permanezca como consecuencia del proyecto.

- Los RESULTADOS son los que el proyecto puede garantizar como consecuencia de sus actividades.

- Las ACTIVIDADES, que son las acciones necesarias para transformar insumos dados en resultados planificados dentro de un período de tiempo dado.

- Los INSUMOS son las "materias primas" de un proyecto para producir los resultados propuestos: medios humanos, técnicos y materiales.

Con esta información los estudiantes abordarán sistemáticamente las problemáticas explicitadas para que desde la EpD se puedan llevar a cabo, haciendo, además del proyecto según el marco lógico, un análisis de la realidad y una justificación - o marco teórico- para el proyecto que lo conecte aún más con el contexto donde se va a desarrollar y un análisis de la sostenibilidad de dicho proyecto. Con esta perspectiva contribuimos, además, a la creación de una conciencia de ciudadanía global en el estudiantado que podrá transmitir en el desarrollo profesional de su carrera. 


\subsection{Las propuestas de trabajo de los estudiantes}

Tal y como se ha puesto de manifiesto en el apartado anterior, cada grupo reducido de estudiantes creaba y gestionaba su propio caso para, posteriormente, buscar una posible solución desde el punto de vista de la educación social. Para algunos autores, como Martínez y Carrasco (2006), cuando se trabaja desde el análisis de un caso, el objetivo principal es motivar hacia el análisis de la situación, ya que se trata de un trabajo de interiorización y reflexión, en este caso particular, realizado en pequeño grupo. De hecho, para Mendoza (2006), el proceso de aprendizaje alcanzado a través del estudio de casos podría considerarse como un aprendizaje significativo, ya que al asociarse a dificultades, los aprendizajes se relacionarían con los conocimientos previos; es participativo, porque el trabajo grupal es necesario para encontrar soluciones; es cooperativo, al implicar a todos los miembros del grupo; facilita aprender a aprender, al favorecer diferentes estrategias cognitivas como respuesta ante las situaciones-problema; motiva hacia la toma de decisiones y de resolución de problemas.

A lo largo de los años que esta asignatura lleva impartiéndose, desde el curso 2012/13 hasta el curso 2017/18, los alumnos y alumnas han propuesto más de 100 proyectos.

Algunos de los proyectos en los que se ha trabajado durante estos años se muestran en el Anexo. Estos proyectos han sido seleccionados de cada curso académico en base a las coevaluaciones del grupo y a la originalidad de la temática y del caso en sí. ${ }^{3}$

\section{Análisis del proceso}

Con el objetivo de determinar aquellos proyectos con mayor repercusión socioeducativa, dentro de cada aula se propuso llevar a cabo un proceso de coevaluación, integrando por un lado, la evaluación procesual del contenido propiamente dicho de los documentos elaborados, la evaluación de grupo a través de una rúbrica de

\footnotetext{
${ }^{3}$ Las variaciones en el número total de proyectos en cada curso es debido a las variaciones en el número de alumnos matriculados y a que en algunos cursos se han realizado actividades paralelas como videos participativos de realidad social mediante la investigación-acción participativa (2012/13: 21 proyectos; 2013/14: 21 proyectos; 2014/15: 27 proyectos; 2015/16: 19 proyectos; 2016/17: 23 proyectos; proyectos; 2017/18: 21 proyectos). Véase: Hermosín-Mojeda, M. J.; Conde-del Río, M. A. y Jiménez Fernández, J. (2016): "El video participativo como herramienta para la asignatura Educación para el Desarrollo". II Congreso Iberoamericano sobre Recursos Educativos Innovadores. Congreso Virtual.
} 
evaluación realizada para la asignatura ad hoc y, por otro lado, a través de las exposiciones realizadas en las últimas sesiones de la asignatura, donde cada grupo de estudiantes debía exponer el proyecto realizado dando a conocer principalmente, la motivación en la selección tanto del país de implementación como de los objetivos planteados, la entidad con la que se asociarían y los posibles resultados. Como se puede deducir, dada la lejanía de los espacios seleccionados, ninguno de los proyectos se ha podido implementar en el periodo de docencia establecido para su elaboración, sin embargo, sí que se solicitaban ítems y evidencias posibles de ser evaluadas para comprobar el grado de consecución de los proyectos.

Los proyectos se exponen y defienden públicamente en el aula con un soporte audiovisual donde cada grupo debe dar a conocer el proceso que ha seguido desde que se selecciona el tema hasta que se plantea el caso a resolver a través de los proyectos. Para que la evaluación sea lo más completa posible, se realiza a varios niveles: autoevaluación -con conciencia crítica del proceso-, análisis de dificultades y propuestas de mejora donde se solicita explícitamente una opción alternativa de volver a comenzar el trabajo en ese momento, es decir, dan cuenta de lo que han aprendido. Se hace heteroevaluación por los compañeros en el momento de su presentación pública. Y, por último, el profesorado evalúa el proyecto realizado a partir de la rubrica elaborada a tal fin.

A través de las temáticas de los proyectos que hemos seleccionado para su análisis podemos ver que el alumnado centra su mirada en realidades concretas de diferentes partes del mundo sin olvidar, claro está, que también pueden hacer educación para el desarrollo en su entorno más cercano. Los colectivos a los que se destinan las propuestas van desde niños con diferentes dificultades (entre los que se incluyen exclusión social y pobreza, por un lado, o enfermedades o discapacidades, por otro), hasta adultos en similares circunstancias o necesitan resocializarse, pasando por colectivos profesionales -educadores- que necesitan formación específica en $\mathrm{EpD}$, ya que esta área no aparece en el currículum de la formación inicial del profesorado y es necesaria si se quiere una escuela que favorezca la ciudadanía global y el desarrollo.

El estudio de los colectivos en los que plantear los proyectos se ha determinado en base a una búsqueda de información exhaustiva, que ha llevado a la mayoría de los grupos a contactar con ONGDs que están trabajando en los diferentes lugares donde se pretende desarrollar dichos proyectos. Para facilitar la aceptación del proyecto y la posibilidad de llevarlo a cabo, en muchos de los proyectos se han establecido puntos de partida de ONGD que ya funcionan, adaptando los planteamientos a los requisitos de los proyectos que llevan a cabo (Intermón-Oxfam, 
Educación Sin Fronteras, InteRed, Muñecas-Madrinas, SOS-mujer, Proyecto de solidaridad de la Facultad de Educación, Psicología y CC. del Deporte de la Universidad de Huelva con el Sáhara, Amnistía Internacional, Cruz Roja, Manos Unidas, ...). Los proyectos, en este caso, deberán incorporar acciones integradoras entre ambas partes, los dos países y las dos instituciones.

Para la búsqueda de financiación de cara a los proyectos planificados, el alumnado selecciona bien un organismo nacional, bien fundaciones $y / o$ empresas que tengan un apartado concreto para la cooperación al desarrollo (AECID, Área de Cooperación Internacional de la Diputación de Huelva, La Caixa, Cepsa,...), adaptándose los proyectos a las bases de las convocatorias y organizando la información en la forma que ellos proponen, en un intento más de contextualizar la forma de trabajo de la vida real.

\section{Resultados y Conclusiones}

Se ha partido de la concepción de que "en la cultura contemporánea, una cuestión fundamental para que un individuo pueda comprender el mundo en el que vive es que sepa cómo acceder, analizar e interpretar la información. En la educación escolar (desde la escuela infantil hasta la universidad) se supone que se ha de facilitar esta serie de competencias, en un proceso que comienza, pero que nunca termina, pues siempre podemos acceder a formas más complejas de dar significado a la información." (Hernández, 2000, p. 46). Es por ello que nuestra primera intención fue la de facilitar un proceso de aprendizaje complementario al propio del currículo académico universitario. Del mismo modo, se ha trabajado para promover en el alumnado la compresión de las situaciones investigadas, con la intención de ser capaces de ir más allá de la información facilitada, reconociendo las necesidades de un hecho y proponiendo soluciones al respecto de manera interdisciplinar, aunque sin perder de vista la respuesta socioeducativa como principal, dentro del ámbito de la educación para el desarrollo y la cooperación internacional.

Para obtener datos concretos sobre si la implantación de la metodología ABP ha supuesto un beneficio al desarrollo de las competencias del alumnado, establecimos un cuestionario de satisfacción respecto a la metodología empleada. En la primera parte se les pidió que valorasen de forma cualitativa diferentes aspectos, tanto de actividades de teoría como de práctica, según los siguientes criterios (mediante valoración con una escala de tipo Likert con cuatro niveles de opinión: 1 -nada de acuerdo- al 4 -totalmente de acuerdo-): 
- Dificultad estimada de la asignatura (contenidos teóricos y prácticos).

- Interés por la actividad (proyecto de cooperación al desarrollo).

- Satisfacción con los resultados obtenidos.

La segunda parte del cuestionario, de tipo cuantitativo, estaba centrada en los resultados (calificaciones obtenidas) en esta asignatura frente a las demás de $3^{\circ}$ de carrera.

Con los resultados obtenidos, que pasamos a comentar a continuación, podemos conocer mejor los puntos fuertes y débiles de esta metodología y así proponer mejoras para mantener la experiencia o bien transformarla en algo que se adecue mejor al perfil del alumnado que cursa esta asignatura.

Las encuestas de satisfacción, que se hicieron mediante Google Forms, no fueron respondidas por la totalidad de los alumnos participantes en la experiencia, pero obtuvimos una muestra lo suficientemente amplia para obtener conclusiones válidas de la efectividad del uso del ABP en la asignatura (489 alumnos/as en total).

En el primer bloque de valoraciones (dificultad estimada de la asignatura) incluimos algunas como las siguientes:

- He experimentado una evolución en mis conocimientos mayor que siguiendo una metodología expositiva o de clases magistrales.

- Los contenidos de la asignatura me han resultado más fáciles de asimilar que en asignaturas similares donde la metodología es más tradicional (clases magistrales)

- Heaprendido contenidos conceptuales y procedimentales que antes desconocía.

- Esta metodología es adecuada para el ámbito universitario.

- Esta metodología me ha permitido poner en práctica lo que estaba aprendiendo en las clases teóricas.

En el segundo bloque de valoraciones (interés por la actividad), algunas de las opciones, entre otras, fueron:

- Esta metodología es adecuada para la asignatura Educación para el Desarrollo y la Cooperación Internacional.

- La metodología ABP ha potenciado y mejorado el trabajo en equipo. 
- El alumnado ha realizado las tareas proporcionalmente entre los miembros del equipo.

- El grado de implicación del alumnado del grupo ha sido satisfactorio.

- El profesorado universitario debería poner más a menudo este tipo de trabajo en sus asignaturas.

- La respuesta del alumnado hacia el proyecto ha sido adecuada.

- El profesorado ha prestado la ayuda adecuada.

- La forma de discusión y planteamiento del proyecto ha sido correcta y ha aportado claridad para elegir la temática.

En el tercer bloque de valoraciones (satisfacción con los resultados), entre otras frases, se han empleado las siguientes:

- Los criterios de evaluación del proyecto han sido adecuados.

- Los ítems de la rúbrica de evaluación eran claros.

- La rúbrica de evaluación fue entregada al inicio del proyecto.

- He aprendido más y mejor con esta metodología que con la que he seguido en otras asignaturas de similar dificultad.

- La autoevaluación me ha ayudado a mejorar mi proceso de trabajo.

- La heteroevaluación ha servido para hacer más justa la evaluación.

- La coevaluación se ha planteado como un modo eficaz de sentirse partícipe de todo el proceso de enseñanza-aprendizaje.

La conclusión principal que extraemos de la valoración del alumnado es que el grado de satisfacción es bastante alto, aunque surgen opiniones contradictorias en algunos aspectos, ya que se permitió a los alumnos que aportaran, además de las valoraciones mediante la escala tipo Likert, que hicieran comentarios cualitativos sobre aspectos que pensaran que no estaban recogidos en dichos ítems.

Esta metodología facilita la comunicación y la interacción de los estudiantes entre sí y con el profesorado, pero la simple implantación no supone buenos resultados si no hay un trabajo continuo de colaboración con el alumnado y supervisión de lo que van haciendo. Es cierto que promueven actitudes que pueden variar desde el rechazo a esta forma de trabajo hasta el entusiasmo, pero es mayor el porcentaje de alumnos que considera que es una mejor forma de aprender y de interiorizar los 
contenidos que la mera lectura de documentos, la clase magistral o la realización de trabajos tipo ensayo donde cada miembro del grupo se dedica a su parte y no hay una verdadera comunicación y compartición del conocimiento.

Haciendo un rápido balance, por bloques de valoraciones, observamos que en el primer bloque (dificultad de la asignatura), ha estado más decantado hacia la percepción de que aprender así les ha resultado más fácil, exceptuando algunos aspectos concretos, como fue la legislación española y del país de destino del proyecto en materia de cooperación internacional, como veremos más adelante. Destaca que el ítem "He experimentado una evolución en mis conocimientos mayor que siguiendo una metodología expositiva o de clases magistrales" obtuvo un $43 \%$ de valoraciones en "totalmente de acuerdo" y un $28 \%$ en "parcialmente en acuerdo", frente a un $29 \%$ que se manifestaba "parcialmente en desacuerdo" o "totalmente en desacuerdo". En cuando al ítem "Esta metodología es adecuada para el ámbito universitario", hemos obtenido unos resultados muy similares, en los que la mayoría estaba de acuerdo o muy de acuerdo. Hacemos constar que había un número importante de estudiantes que no había experimentado el $A B P$ en sus etapas educativas previas a la universidad, por lo que es importante destacar que para un $78 \%$ esta era una forma de trabajo totalmente novedosa.

En el segundo bloque de valoraciones (interés por la actividad), los resultados nos llevan a pensar que esta forma de trabajo ha suscitado, claramente mayor interés por los contenidos que con otras metodologías más tradicionales. De tal forma que en el ítem "La metodología ABP ha potenciado y mejorado el trabajo en equipo", más del $65 \%$ del alumnado se encontraba "totalmente de acuerdo" ○ "parcialmente de acuerdo", frente a un $7 \%$ que se manifestaba "totalmente en desacuerdo". El ítem "El grado de implicación del alumnado del grupo ha sido satisfactorio" ha sido muy bien valorado en general $y$, si lo miramos desde el punto de vista del profesorado -a través la observación del trabajo en clase de los grupos mediante una lista de cotejo-, ha sido igualmente significativa, ya que se ha podido comprobar el trabajo diario en las sesiones de clase.

Existe un amplio consenso entre el alumnado cuando se le pide opinión sobre si "El profesorado universitario debería poner más a menudo este tipo de trabajo en sus asignaturas", pues el 64\% está "totalmente de acuerdo" y el 32\% está "parcialmente de acuerdo". Por lo que consideramos que "La respuesta del alumnado hacia el proyecto ha sido adecuada", como así lo manifiestan ellos mismos.

En el tercer bloque de valoraciones (satisfacción con los resultados) -que se complementa con un análisis cuantitativo en el que comparamos las calificaciones de los 
alumnos y alumnas en $3^{\circ}$ de carrera-, observamos que en el ítem "He aprendido más y mejor con esta metodología que con la que he seguido en otras asignaturas de similar dificultad", hay un $52 \%$ que está totalmente de acuerdo y un $27 \%$ que está "parcialmente de acuerdo". Y en cuando a los ítems relacionados con la forma en la que se ha efectuado la evaluación-autoevaluación, heteroevaluación y coevaluación-, en general, les ha hecho sentirse protagonistas de dicho proceso, como manifiestan cuando un $44 \%$ responde sentirse "totalmente de acuerdo" y un $32 \%$ expresa que se siente "parcialmente de acuerdo" por lo que solo un $24 \%$ estaría situado entre el "parcialmente en desacuerdo" $\mathrm{y}$ "totalmente en desacuerdo" respecto el ítem "La coevaluación se ha planteado como un modo eficaz de sentirse partícipe de todo el proceso de enseñanza-aprendizaje".

Aunque en líneas generales podemos afirmar que los resultados son muy satisfactorios, el alumnado se ha encontrado con algunas dificultades a la hora de la resolución de los proyectos y nos lo han hecho saber a través de los espacios para opinar libremente en cada uno de los apartados:

- Desconocimiento de la legislación internacional del país seleccionado en el caso de proyectos internacionales y dificultad para encontrar información clara al respecto.

- Elaboración de presupuestos realistas y fórmulas de financiación.

- Diseño de objetivos operativos claros y cuantificables, así como las formas de evaluarlos, es decir, trazabilidad entre los objetivos, actividades que lo desarrollan, criterios de evaluación e instrumentos para recoger evidencias y momentos de evaluación.

- Confección de la matriz del proyecto según el marco lógico: árbol de problemas y árbol de soluciones.

- Estructura jurídica de las ONGD.

- Compromisos de la parte y la contraparte.

Para intentar paliar algunos de estos inconvenientes y reducir las dificultades, fruto de las evaluaciones de esta metodología, a partir del primero, cada nuevo curso, con el comienzo de la asignatura se organiza una mesa redonda con miembros activos de ONGDs con el objetivo de que cuenten cómo inician y llevan a cabo sus proyectos y que los estudiantes puedan preguntar sus dudas y aclarar algunos puntos que la teoría simplifica sobremanera. Igualmente se cuenta con profesorado de la Facultad que participan activamente en diversos proyectos de cooperación internacional. 
Desde un punto de vista más cuantitativo, ofrecemos las siguientes gráficas que responden a una recogida de datos sobre los resultados obtenidos en esta asignatura comparados con el resto de las asignaturas de $3^{\circ}$ del Grado en Educación Social, donde predominan las de carácter obligatorio:

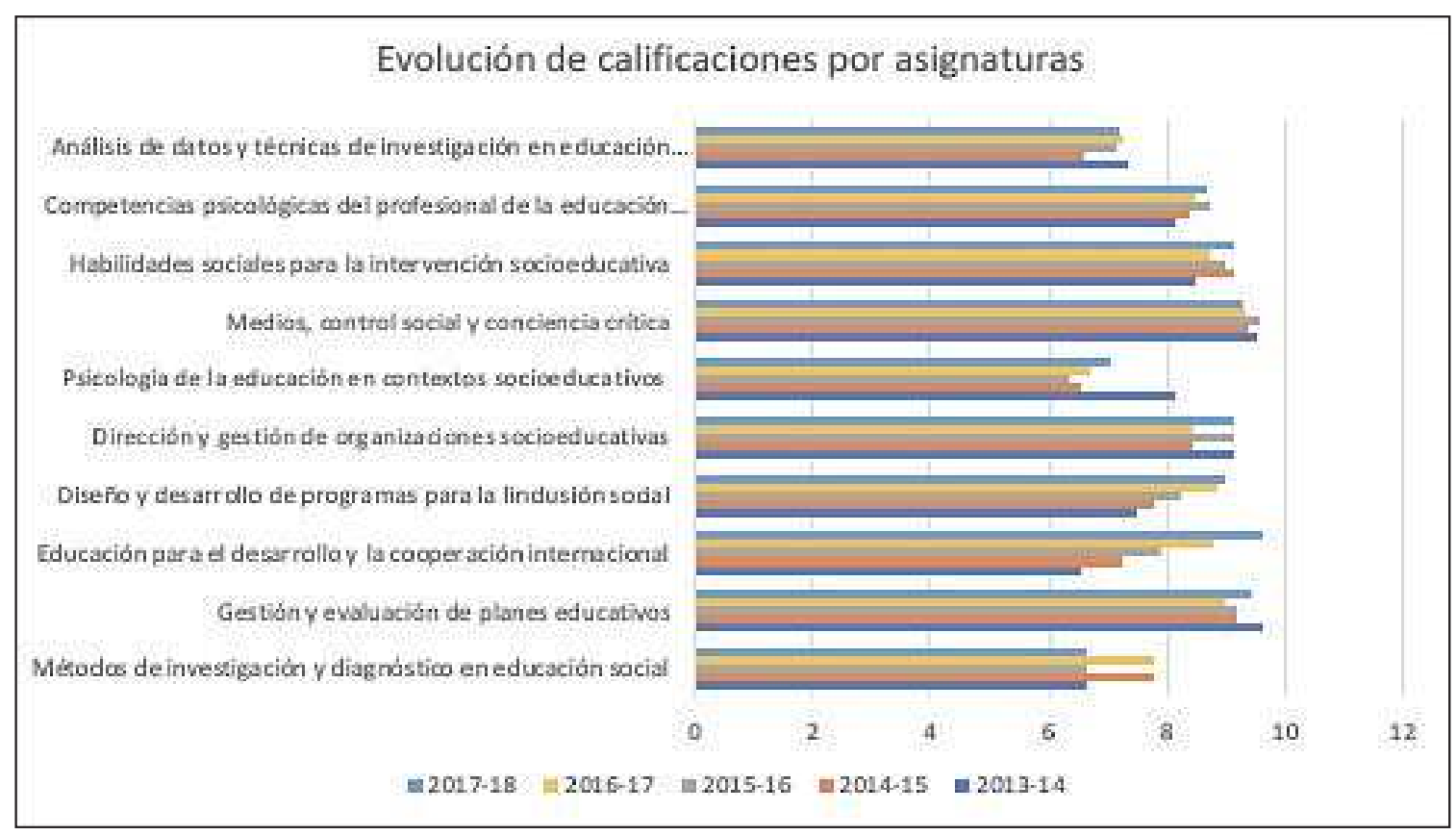

Según esta gráfica en la que se comparan las asignaturas obligatorias de $3^{\circ}$ y las dos optativas con mayor número de matriculados (Psicología de la Educación en contextos Socioeducativos y Habilidades Sociales para la intervención Socioeducativa), observamos que la evolución en esta asignatura es muy positiva a lo largo de estos cursos, pasando de una media de aprobado por encima de 6 puntos hasta llegar, al final de los años que hemos tenido en cuenta, a alcanzar una media de sobresaliente. Esto es debido, quizá a que los contenidos cuando han sido expuestos siguiendo una metodología más tradicional, el alumno se ha visto menos implicado y los contenidos no han sido tan bien asimilados por su parte, mientras que a medida que se ha cambiado la metodología al $A B P$, el interés ha aumentado y, consecuentemente, la implicación y los resultados.

Entresacando los datos de la asignatura, obtenemos la siguiente gráfica: 


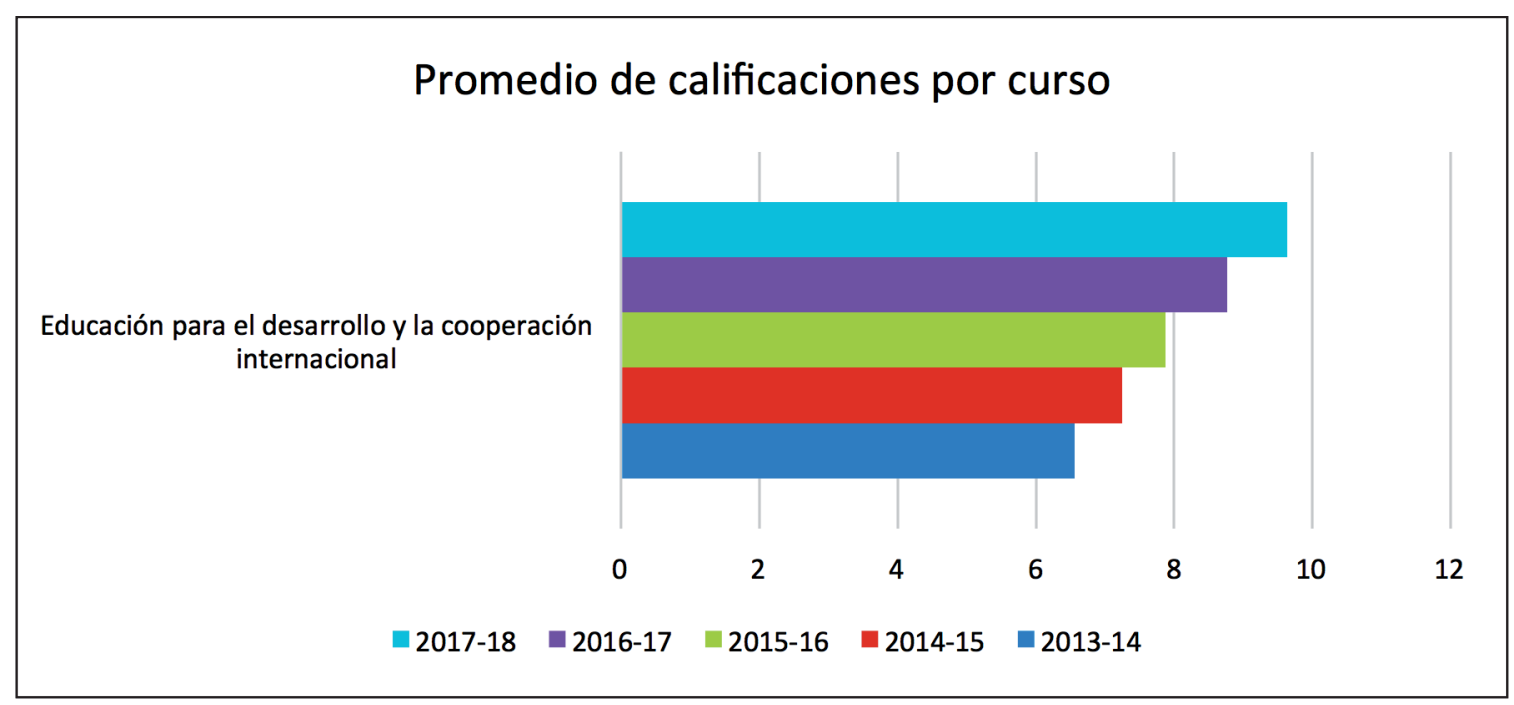

A pesar de la evolución favorable y las opiniones en ese mismo sentido del alumnado, no todo es positivo, porque trabajando de esta manera se produce una mayor carga de trabajo para el profesorado, dado el elevado número de alumnos que necesita una importante dedicación a la gestión de las tareas dentro del aula-seguimiento y asesoramiento mientras están haciendo los proyectos- como fuera-tutorías grupales obligatorias-.

No obstante, valoramos satisfactoriamente los resultados obtenidos tras varios años de aplicación de esta metodología porque se ha logrado que gran parte de los estudiantes desarrollen aprendizajes más cercanos a su futura práctica profesional, sustituyendo en gran parte el estudio memorístico previo al examen, por una actividad continuada, mantenida a lo largo de la asignatura, que genera aprendizajes más profundos.

Con todo lo expuesto podemos afirmar, finalmente, que el trabajo por proyectos en nuestra asignatura aporta los elementos necesarios para que los estudiantes den un paso adelante en la creación de la ciudadanía global desde la vivencia propia y logren establecer pautas para favorecer la EpD. Los educadores y educadoras sociales son agentes de transformación social, elementos clave para articular proyectos de cambio de realidad próxima, o tratar de mejorar la que es desfavorable en otras partes del mundo gracias a la intervención. Somos conscientes de las limitaciones existentes en la estructura rígida de la academia para integrar saberes prácticos a las aulas universitarias; sin embargo, en los últimos años se está haciendo un esfuerzo desde el profesorado para permitir la integración de conocimientos que faciliten el desarrollo de competencias profesionales más allá de las paredes de la institución. 


\section{Referencias}

Almagro, A. y Pérez, M. L. (2004). "Making the case method work in teaching Business English: a case of study". English for Specific Purposes, 23, 137-161.

Alonso Martín, P.; Cruz Díaz, R. y Hermosín-Mojeda, M. J. (2010). "Análisis del nivel de desarrollo e importancia de las competencias transversales en el alumnado de la Facultad de CC. de la Educación para la mejora de la calidad docente", Actas del VI Congreso Internacional de Docencia Universitaria e Innovación (VI CIDUI), Nuevos Espacios de Calidad en la Educación Superior. Un análisis comparado de tendencias, Barcelona, Universitat Politècnica de Catalunya.

BEL, S. (2010). Project-based learning for the $21^{\text {st }}$ century: Skills for the future. The Clearing House, 83 (2), 39-43. doi: 10.1080/00098650903505415

DelGADO, J. M. y GUTIÉRREZ, J. (1999). Métodos y técnicas cualitativas de investigación en ciencias sociales. Madrid: Síntesis.

FeRNÁNDEZ, A. (2006). "Metodologías activas para la formación de competencias". Educatio Siglo XXI, 24, 35-56.

FERRERO Y DE LOMA-OSORIO, G. (ed.) (2008). Identificación y formulación de proyectos de cooperación para el desarrollo: gestión del ciclo del proyecto y enfoque del marco lógico, Valencia, Universidad Politécnica de Valencia.

Gómez Galán, M. y Sainz Ollero, H. (2013). El ciclo del proyecto de cooperación al desarrollo. La aplicación del marco lógico, Madrid, CIDEAL.

Hernández, F. (2000). "Los proyectos de trabajo; la necesidad de nuevas competencias para nuevas formas de racionalidad". Educar, 26, 39-51

Kraucik, J. S. y Blumenfeld, P. C. (2006). "Project based learning", en SaWyer, R. K. (ed) The Cambridge Handbook of the Learning Sciences, Cambridge, Cambridge University Press.

Martínez, M. y Carrasco, S. (coords.) (2006). Propuestas para el cambio docente en la universidad. Barcelona: Octaedro.

MendozA, A. (2006). El estudio de casos. Un enfoque cognitivo. Trillas: Sevilla. 
Mérida, R., Barranco, B., Criado, E., Fernández, N., López, R. M. y Pérez, I. (2011). "Aprender investigando en la escuela y en la universidad. Una experiencia de investigación-acción a partir del Trabajo por Proyectos". Investigación en la Escuela, 73, 65-76.

Navarro Montaño, M. J. (201 1). "Metodologías activas y participativas en educación superior. Estudio de casos". Enseñanza, 261-293

Orejudo Hernández, S., Fernández Turrado, T. y Garrido laparte, Mª. A. (2008). "Elaboración y trabajo con casos y otras metodologías activas. Cuatro experiencias de un grupo de profesores de la facultad de educación de Zaragoza". Revista Interuniversitaria de Formación del Profesorado, 63 (22,3), 21-45.

Ortega Carpio, M. L., Sianes Castaño, A. y Cordón Pedregosa, M. R. (2012). "La educación para el desarrollo en la lucha contra la pobreza: aportes para el profesorado universitario" , Revista de Fomento Social, Córdoba, ETEA, n. 67, 607-633.

Palomero, J. E. (Coord.) (2003). "Enseñar y aprender en la universidad". Revista Interuniversitaria de Formación del Profesorado, 47, pp. 15-136.

Parrilla latas, A., Muñoz-Cadavid, M. A. y Sierra Martínez, S. (2013). "Proyectos educativos con vocación comunitaria". Revista de Investigación en Educación, 11 (3), pp. 15-31.

Rodríguez, A. B., Ramírez, L. J. y Fernández, W. (2017). Metodologías activas para alcanzar el comprender. Formación Universitaria, vol. 10(1), 79-88. http://dx.doi. org/10.4067/S0718-50062017000100009

SIEUPM (2008). Aprendizaje orientado a proyectos. Guías rápidas sobre nuevas metodologías, Madrid, Universidad Politécnica de Madrid.

Vergara Ramírez, J. J. (2014). "10 claves para trabajar la Educación para el Desarrollo (EpDCG) por el Método de Proyectos (ABP)", Revista Internacional Sobre Investigación en Educación Global y para el Desarrollo, Madrid, FERE-CECA, n. $5,148-164$.

VILAR, S. (1997). La nueva racionalidad. Comprender la complejidad con métodos transdisciplinarios. Barcelona, Kairós. 


\section{ANEXO. Selección de proyectos realizados por el alumnado}

\section{TABLA I. Proyectos seleccionados Cursos 2012-13 a 2017-18 (total de proyectos: 123)}

\begin{tabular}{|c|c|c|}
\hline Título & Colectivo & Objetivos del proyecto (2012/13) \\
\hline $\begin{array}{l}\text { Educación de niños } \\
\text { en Rumanía (etnia } \\
\text { gitana). }\end{array}$ & $\begin{array}{l}\text { Niños de etnia gita- } \\
\text { na en la Escuela rural } \\
\text { de Ploiesti (Prahova } \\
\text { - Rumanía). }\end{array}$ & $\begin{array}{l}\text { Mejorar la escolarización obligatoria de los } \\
\text { niños gitanos en Rumanía. } \\
\text { - Crear personal cualificado para la forma- } \\
\text { ción de los profesionales. } \\
\text { - Mejorar la intermediación entre familias } \\
\text { y escuela. } \\
\text { - Motivar a los alumnos sobre la importancia } \\
\text { de la educación secundaria. }\end{array}$ \\
\hline Reconstrúye-T. & $\begin{array}{l}\text { Inmigrantes desem- } \\
\text { pleados en Lorca } \\
\text { (Murcia). }\end{array}$ & $\begin{array}{l}\text { Mejorar las condiciones de vida de los inmi- } \\
\text { grantes a través del empleo. } \\
\text { - Enseñar estrategias para una búsqueda } \\
\text { eficaz de empleo. } \\
\text { - Entrenar estrategias para afrontar entre- } \\
\text { vistas de trabajo. }\end{array}$ \\
\hline Título & Colectivo & Objetivos del proyecto $(2013 / 14)$ \\
\hline Mujeres olvidadas. & $\begin{array}{l}\text { Viudas menores de } \\
18 \text { años en Vrinda- } \\
\text { van (India). }\end{array}$ & $\begin{array}{l}\text { Formar e informar al colectivo de mujeres } \\
\text { olvidadas (viudas de la India) sobre diversas } \\
\text { temáticas. } \\
\text { - Formar a ese colectivo en herramientas } \\
\text { para la búsqueda de empleo. } \\
\text { - Formar para el autoempleo. }\end{array}$ \\
\hline $\begin{array}{l}\text { "Muñecas madri- } \\
\text { nas": un corazón } \\
\text { de trapo. }\end{array}$ & $\begin{array}{l}\text { Sociedad en gene- } \\
\text { ral. (Almonte, Huel- } \\
\text { va, España). }\end{array}$ & $\begin{array}{l}\text { Crear nuevos recursos tecnológicos para la } \\
\text { ONG "Muñecas madrinas" (perfil de Face- } \\
\text { booky un blog) donde darse a conocer ante la } \\
\text { sociedady difundir la obra social que realizan. } \\
\text { - Aumentar la colaboración de voluntarios y } \\
\text { la participación del pueblo donde radica } \\
\text { la ONG. } \\
\text { - Mantener actualizados los recursos tecno- } \\
\text { lógicos que se ofrecen a la ONG. }\end{array}$ \\
\hline
\end{tabular}




\section{Título}

Voluntariado, un estilo de vida.

'Passe-moi la bole'.

\section{Título}

Familias magrebíes con niños de 6 a 12 años con discapacidad intelectual en riesgo de exclusión social.

Infancia: $6-12$ años y familias (Marruecos).

\section{Título}

Prevención del consumo de sustancias tóxicas durante la gestación en Ciudad de México.

Nuevas capacidades. Mayores con menores con discapacidad intelectual a su cargo.

\section{Colectivo}

Comunidad de la ciudad en general (Finlandia).

Infancia-adolescencia: 10-16 años (Lyon, Francia).

\section{Colectivo}

Familias magrebíes con niños de 6 a 12 años con discapacidad intelectual en riesgo de exclusión social.

Infancia: 6-12 años y familias (Marruecos).

\section{Colectivo}

Mujeres jóvenes e mbarazadas (México).

65-70 años (Italia).

\section{Objetivos del proyecto $(2014 / 15)$}

Sensibilizar a la población en general de la necesidad de apoyo a los niños hospitalizados 7-15 años.

- Fomentar acciones para el voluntariado en hospitales mediante acuerdoy colaboración con la dirección.

- Realizar talleres de formación para voluntarios.

Desarrollar talleres de formación en valores (Interculturalidad) a través del deporte.

- Elegir valores con el grupo para trabajarlos por meses.

\section{Objetivos del proyecto $(2015 / 16)$}

Familias magrebíes con niños de 6 a 12 años con discapacidad intelectual en riesgo de exclusión social.

Infancia: 6-12 años y familias (Marruecos)

\section{Objetivos del proyecto $(2016 / 17)$}

Sensibilizar a la población sobre consecuencias del consumo de sustancias tóxicas durante la gestación y mejora de la calidad de vida.

Mejorar la calidad de vida, en el ámbito de las relaciones de las personas mayores 


\begin{tabular}{|c|c|c|}
\hline Título & Colectivo & Objetivos del proyecto $(2017 / 18)$ \\
\hline \multirow[t]{4}{*}{$\begin{array}{l}\text { Prevención VIH/ } \\
\text { SIDA. }\end{array}$} & \multirow{4}{*}{$\begin{array}{l}\text { Jóvenes de } 18 \text { a } \\
21 \text { años del barrio } \\
\text { Las Palmas (Guinea } \\
\text { Ecuatorial). }\end{array}$} & $\begin{array}{l}\text { Incrementar o mejorar los conocimientos sobre } \\
\text { el VIH/SIDA. }\end{array}$ \\
\hline & & $\begin{array}{l}\text { Prevenir las enfermedades de transmisión } \\
\text { sexual y el VIH. }\end{array}$ \\
\hline & & $\begin{array}{l}\text { - Fomentar hábitos de conducta saludable } \\
\text { para prevenir el contagio por VIH/SIDA. }\end{array}$ \\
\hline & & $\begin{array}{l}\text { - Elaborar un programa formativo (taller) } \\
\text { para dar a conocer las vías de transmisión } \\
\text { del VIH y cómo prevenirlo. }\end{array}$ \\
\hline \multirow[t]{3}{*}{ Aulas hospitalarias. } & \multirow[t]{3}{*}{$\begin{array}{l}\text { Periodo escolar pri- } \\
\text { maria (Portugal). }\end{array}$} & $\begin{array}{l}\text { Proporcionar atención educativa al alumnado } \\
\text { hospitalizado. }\end{array}$ \\
\hline & & $\begin{array}{l}\text { - Llevar a cabo talleres de habilidades } \\
\text { sociales. }\end{array}$ \\
\hline & & $\begin{array}{l}\text { - Realizar talleres de formación del } \\
\text { voluntariado. }\end{array}$ \\
\hline
\end{tabular}

Fuente: Elaboración propia. 